\title{
Estudio longitudinal de la escritura en estudiantes con Trastorno por Déficit de Atención con Hiperactividad (TDAH)
}

\author{
Eva SANZ DOMÍNGUEZ \\ Pilar MARTÍN-LOBO \\ José David URCHAGA LITAGO \\ María Jesús IRURTIA MUÑIZ
}

\author{
Datos de contacto: \\ Eva Sanz Domínguez \\ Universidad de Valladolid \\ evasad@hotmail.com \\ Pilar Martín-Lobo \\ Universidad Internacional de la \\ Rioja \\ pmartinlobo@gmail.com \\ José David Urchaga Litago \\ Universidad Pontificia de \\ Salamanca \\ jdurchagali@upsa.es \\ María Jesús Irurtia Muñiz \\ Universidad de Valladolid \\ mjirurtia@uva.es
}

Recibido: 20/02/2020 Aceptado: 26/06/2020
En la presente investigación se ha realizado una evaluación de la escritura en estudiantes con diagnóstico de Trastorno por Déficit de Atención con Hiperactividad (TDAH). Para ello, se analizan las diferencias en el desarrollo de la escritura en sujetos con TDAH a lo largo de 5 años, comparándolas con otros estudiantes sin diagnóstico de TDAH. Los participantes son 24 sujetos clasificados en dos grupos, con y $\sin$ TDAH, siendo evaluados a través de subpruebas del Test de Análisis de lectura y escritura (TALE) en el año 2014 y 5 años después, 2019. Los resultados muestran, en aquellos participantes sin TDAH, que a medida que mantienen la escolarización su grafismo tiende a mejorar. Por otra parte, aquellos estudiantes con TDAH muestran mejoría únicamente en el tamaño de las letras y en la interlineación. Por lo tanto, se evidencia que, 5 años después, este colectivo mejora menos en el grafismo $y$, resulta necesario realizar algún programa de intervención rehabilitadora en esta población.

PALABRAS CLAVE: escritura; grafismo; trastorno por déficit de atención con hiperactividad; TDAH. 


\title{
Longitudinal study of handwriting in students with disorders for hyperactivity attention deficit (ADHD)
}

\begin{abstract}
In the present investigation, we have been carry out an evaluation of the writing in students with a diagnosis of Attention Deficit Hyperactivity Disorder (ADHD). For this, we have been analysed the differences in the development of writing in students with ADHD, 5 years later, comparing them with other students without a diagnosis of ADHD. The participants are 24 children classified in two groups, with and without ADHD, they have been evaluated through subtests of the Reading and Writing Analysis Test (Spanish: TALE) in 2014 and 5 years later, 2019. The results show, in those participants without ADHD, that as they maintain schooling their graphics of the letters tend to improve. On the other hand, those students with ADHD show improvement only in the size of the letters and in the line spacing. Therefore, it's evident that, 5 years later, this group improves less on the graphics of the letters and, it's necessary to carry out some rehabilitation intervention program with them.
\end{abstract}

KEYWORDS: writing; graphics; attention deficit hyperactivity disorder; ADHD.

\section{Introducción}

Las investigaciones en neurociencia aplicada a la educación muestran la importancia de aprovechar las etapas escolares para desarrollar el potencial de cada estudiante, puesto que el desarrollo neurológico que se da en esas edades facilita el aprendizaje a nivel superior (Szucs y Goswami, 2007). Las investigaciones de Merzenich y su equipo han permitido argumentar que practicar una destreza nueva en las condiciones adecuadas, es decir, teniendo en cuenta las leyes de la plasticidad cerebral, puede cambiar numerosas conexiones entre las células nerviosas y el cerebro (Merzenich, Tallal, Peterson, Millar, y Jenkins, 1999 citado en Alcover y Rodríguez-Mazo, 2012) y puede mejorar las condiciones de aprendizaje de los estudiantes con dificultades educativas y trastornos como la dislexia y el TDAH (Ortiz, 2009); desarrollando las habilidades y destrezas para un razonamiento complejo, mejorando las funciones cognitivas y el rendimiento escolar (Betegón, Rodríguez-Medina, e Irurtia, 2019; Howard-Jones, 2011; Martin-Lobo, 2016; Marambio, 2017). Las técnicas instrumentales básicas del aprendizaje, como son la lectura, la escritura y el aprendizaje matemático, se pueden beneficiar de estos avances y, especialmente en el caso de la escritura, se pueden mejorar los procesos neuropsicológicos implicados como, por ejemplo, los motores, perceptivo visuales, cinestésicos, 
auditivos y de conciencia fonológica (Rigal, 2006). De este modo, se convertirán los grafemas en fonemas y procesos cognitivos de atención y memoria, que sirvan para recordar el orden correcto de las letras o palabras durante su ejercicio, antes de que el correspondiente plan motor sea seleccionado y ejecutado (Volman, Schendel, y Jongmans, 2006). Algunas investigaciones indican que los alumnos con TDAH presentan limitaciones en el autocontrol emocional y en el desarrollo y ejecución de las habilidades de autorregulación (Barkley y Murphy, 2010) y, en consecuencia, pueden mostrar dificultades de aprendizaje, específicamente en la escritura.

El TDAH es un trastorno de dificultades atencionales que se ha incrementado notablemente en los estudiantes de edades correspondientes a la infancia y adolescencia (Catalá-López y Hutton, 2018). Según la American Psychiatric Association, el TDAH es un trastorno del neurodesarrollo cuya característica principal es un patrón persistente de inatención y/o hiperactividad e impulsividad que interfiere en el funcionamiento o en el desarrollo del alumnado, siendo en niños un 5\% y en niñas un 2,5\% (APA, 2014).

La atención es necesaria para realizar los procesos de aprendizaje, puesto que requieren concentración e implicación activa en la tarea (Razza, Martin, y Brooks-Gunn, 2012) y los estudiantes que tienen dificultades atencionales suelen presentar dificultades también en el ámbito escolar (Sánchez-Pérez y González-Salinas, 2013); junto con manifestaciones conductuales de irritabilidad, baja tolerancia a la frustración y estado de ánimo cambiante. Todo ello, incide en dificultades de aprendizajes básicos como la lecto-escritura, que en personas con TDAH supone un $50 \%$ de población con problemas en esta habilidad (Fernández-Jaén et al., 2018). En concreto, según Mayes, Breaux, Calhoun y Frye (2017), la disgrafia entendida como un trastorno del aprendizaje que se manifiesta en una alteración en el proceso de la escritura, está presente en la mitad de los escolares con TDAH de presentación combinada.

La escritura es una tarea que requiere procesos neuropsicológicos cerebrales de atención y de motricidad en los que están implicadas también habilidades visuales (motricidad, acomodación y convergencia visual entre otras), junto a habilidades motrices y de coordinación visomotriz, para lograr que la mano pueda ejecutar las acciones propias de la escritura (Díaz-Jara, 2016). Existen diferentes circuitos y redes neuronales que realizan las funciones cerebrales de la atención, de la motricidad y de la escritura los cuales son la base neuropsicológica para que los sujetos puedan escribir de forma eficiente (Tabla 1). 
Tabla 1

Órgano funcional de la escritura (Quintanar y Solovieva, 2003, citado en Cisneros, Lázaro, Garcia, Garcia y Quintanar, 2012, p.57)

\begin{tabular}{|c|c|c|c|}
\hline Eslabón & Factor & Zona cerebral & Función \\
\hline $\begin{array}{l}\text { Imagen visual de } \\
\text { la letra }\end{array}$ & $\begin{array}{l}\text { Retención } \\
\text { visual }\end{array}$ & Occipitales & Análisis de los elementos \\
\hline $\begin{array}{l}\text { Imagen visuo- } \\
\text { espacial }\end{array}$ & $\begin{array}{l}\text { Perceptivo } \\
\text { global } \\
\text { Perceptivo } \\
\text { analitico }\end{array}$ & $\begin{array}{l}\text { Temporoparieto- } \\
\text { occipitales }\end{array}$ & $\begin{array}{l}\text { Diferenciación de letras } \\
\text { similares }\end{array}$ \\
\hline \multirow[t]{2}{*}{$\begin{array}{l}\text { Ejecución de la } \\
\text { escritura }\end{array}$} & Cinestésico & Parietal & $\begin{array}{l}\text { Esquema de movimientos } \\
\text { de acuerdo a la imagen }\end{array}$ \\
\hline & Cinético & $\begin{array}{l}\text { Frontal } \\
\text { posterior }\end{array}$ & $\begin{array}{l}\text { Movimientos finos, pasos } \\
\text { fluentes de un elemento a } \\
\text { otro }\end{array}$ \\
\hline Intención & $\begin{array}{l}\text { Regulación } \\
\text { y control }\end{array}$ & $\begin{array}{l}\text { Lóbulos } \\
\text { frontales }\end{array}$ & $\begin{array}{l}\text { Objetivo, elección del } \\
\text { programa de } \\
\text { combinaciones de letras, } \\
\text { control de la } \\
\text { comprensión del sentido } \\
\text { y de los signos de } \\
\text { puntuación }\end{array}$ \\
\hline $\begin{array}{l}\text { Correlación } \\
\text { entre sonido y } \\
\text { letra a través de } \\
\text { la pronunciación }\end{array}$ & Cinestésico & Parietal & $\begin{array}{l}\text { Diferenciación de } \\
\text { articulemas cercanos }\end{array}$ \\
\hline $\begin{array}{l}\text { Percepción de } \\
\text { sonidos }\end{array}$ & $\begin{array}{l}\text { Oído } \\
\text { fonemático }\end{array}$ & $\begin{array}{l}\text { Temporal } \\
\text { superior }\end{array}$ & $\begin{array}{l}\text { Diferenciación de } \\
\text { fonemas (vocal- } \\
\text { consonante, sordos- } \\
\text { sonoros, etc.) }\end{array}$ \\
\hline $\begin{array}{l}\text { Memoria audio- } \\
\text { verbal }\end{array}$ & $\begin{array}{l}\text { Retención } \\
\text { audio- } \\
\text { verbal }\end{array}$ & $\begin{array}{l}\text { Temporal } \\
\text { amplia }\end{array}$ & Material para la escritura \\
\hline
\end{tabular}

Autores como Van Hoorn, Maathuis, Peters y Hadders-Algra (2010) indican que las condiciones neurológicas juegan un papel importante. Adi-Japha et al. (2007) sugieren que los problemas en la escritura en el TDAH están asociados con problemas atencionales que reflejan una dificultad en los procesos motores. Otros estudios argumentan que es común que este trastorno coexista con déficits grafomotores asociados con dificultades de coordinación y de control motor fino, necesarios para el trazado de las letras (Abad-Mas, Ruíz-Andrés, Moreno-Madrid, Herrero-Lladró, y Suay-Nicolás, 2013; Lipowska, 2012; Stasik, Tucha, Tucha, Walitza, y Lange, 2009).

Además de estas habilidades neuropsicológicas, el proceso de la escritura exige múltiples recursos cognitivos superiores que pueden resultar dificiles en los estudiantes con TDAH, debido, sobre todo, a la 
dificultad que tienen para inhibir la impulsividad y mantener la atención (Rodríguez et al., 2010). El escritor efectivo selecciona, adopta o inventa estrategias que mejoren sus objetivos, es decir, escribe de forma autorregulada (García-Sánchez y Rodríguez-Pérez, 2007); este proceso que exige reflexión y es difícil llevarlo a cabo en casos de TDAH (Barkley, 2006) supone mayor esfuerzo, escritura de menor calidad e inferior rendimiento académico general (Montoya-Londoño, VarelaCifuentes, y Dussan-Lubert, 2012). Además, algunos estudios muestran que estos estudiantes tienen pobre coordinación motora fina manifestando problemas para pintar, cortar con tijeras, amarrar los cordones de los zapatos y abotonar (Barckley y Murphy, 2005 citado en Rivera-Flores, 2013) y un movimiento exagerado que incidirá en la forma de grafiar las letras de forma precipitada con alteraciones gráficas.

La madurez general del sistema nervioso y el nivel de desarrollo motor actúan sobre el tono muscular y la coordinación fina de los dedos y de la mano, dando lugar a tres etapas en el aprendizaje gráfico: 1) la primera etapa, denominada precaligráfica, corresponde a los inicios de la escritura hasta los 8 años, donde el trazo comienza a ser más seguro y organizado, a través de la repetición del movimiento, pasando del control visual al control cinestésico; 2) este hecho da lugar a la segunda fase, llamada caligráfica y que dura hasta los 13 años, en la que el trazo denota una mayor organización de movimiento, el tamaño de las letras se normaliza y las líneas son rectas y espaciadas; 3) hasta llegar, por último, a la fase postcaligráfica, después de los 13 años, donde se personaliza la forma e inclinación de las letras y donde las demandas de rapidez hacen que pase a un segundo plano la calidad del trazo.

Según Graham, Weintraub, y Berninger (2006), con la edad, la legibilidad y la velocidad de la escritura progresan; la formación de la letra, el espacio y el alineamiento tienden a ser más exactos; la escritura es más abreviada y la letra cursiva o ligada se desarrolla con curvas que reemplazan a los ángulos y a las ligaduras que unen a las letras y, finalmente, el tamaño de la letra tiende a disminuir.

Son escasas las investigaciones que examinan el origen, la prevalencia y los determinantes que influyen en el déficit de la escritura en niños con TDAH. Sin embargo, algunas de ellas se han focalizado en aspectos cualitativos de la letra como el espaciado, basándose en el criterio subjetivo del examinador (Martín Fernández-Mayoralas et al., 2013). Asimismo, estudios comparativos, señalan la necesidad de realizar nuevos estudios y programas sobre la escritura en este colectivo, aportando nuevos conocimientos y vías para la intervención y la mejora de este aprendizaje (Rodríguez et al., 2009). 


\section{Método}

\section{Objetivo / Hipótesis}

El objetivo es estudiar si niños de Educación Primaria (EP) tienen la misma evolución en la escritura con el paso del tiempo en función de si padecen o no TDAH.

La hipótesis planteada es que los estudiantes de EP sin TDAH mostrarán mejor evolución en la calidad de su escritura, al cabo de cinco años, frente a los que tengan dicho diagnóstico.

\section{Muestra}

Para la selección de la población se ha seguido un procedimiento no probabilístico, en concreto un muestreo a conveniencia, formado finalmente por 9 estudiantes sin TDAH y 15 con TDAH; además ninguno de los participantes presenta otro diagnóstico. Los sujetos están escolarizados entre $2^{\circ}$ y $6^{\circ}$ curso de EP en 6 colegios públicos, así como en asociaciones de niños con TDAH de la comunidad de Castilla y León. Es importante señalar que se elige la etapa de EP, por ser el periodo de tiempo en el que se automatiza el proceso de la escritura descartando la edad de 6 años, pues se trata de un período de iniciación en esta habilidad.

La composición de los grupos se lleva a cabo utilizando sujetos que difieren en tener o no un diagnóstico de TDAH. El criterio de inclusión de los alumnos con diagnóstico de TDAH, ha sido el haber sido evaluados por el Equipo de Orientación Educativa y Psicopedagógica (EOEP) del centro educativo, además de estar incluidos en el fichero automatizado "Datos de carácter personal relativos al alumnado con Necesidades Educativas Específicas" ATDI mediante la Orden EDU/571/2005 de 26 de abril en el Grupo ACNEE (alumnado con necesidades educativas especiales) en la Tipología Trastornos por déficit de atención y comportamiento perturbador y en la Categoría TDAH.

La media de edad del Grupo con TDAH al finalizar el estudio fue de $M=14.40$ (DT: 1.6) y la del grupo sin TDAH de $M=14.67$ (DT: 1.8), no siendo significativa la diferencia de edad entre ambos grupos (prueba t, sig.: .709) (Tabla 2). 
Tabla 2

Distribución de participantes por edad

\begin{tabular}{|c|c|c|c|c|}
\hline \multirow{3}{*}{ Edad al seguimiento } & \multicolumn{3}{|c|}{ Diagnóstico } & \\
\hline & \multicolumn{2}{|c|}{ Con TDA/TDAH } & \multicolumn{2}{|c|}{ Sin TDA/TDAH } \\
\hline & $\mathrm{n}$ & $\%$ & $\mathrm{n}$ & $\%$ \\
\hline 11 años & 1 & $6.7 \%$ & 1 & $11.1 \%$ \\
\hline 13 años & 2 & $13.3 \%$ & 1 & $11.1 \%$ \\
\hline 14 años & 7 & $46.7 \%$ & 1 & $11.1 \%$ \\
\hline 15 años & 1 & $6.7 \%$ & 3 & $33.3 \%$ \\
\hline 16 años & 2 & $13.3 \%$ & 2 & $22.2 \%$ \\
\hline 17 años & 2 & $13.3 \%$ & 1 & $11.1 \%$ \\
\hline Total & 15 & $100 \%$ & 9 & $100 \%$ \\
\hline
\end{tabular}

\section{Diseño}

El estudio se realiza desde un enfoque cuantitativo. Para ello, se ha elegido un diseño con medidas repetidas en dos momentos de evaluación pre y post, con una diferencia de 5 años entre las dos mediciones (2014-2019). La evaluación se realiza en ambos grupos (población con y sin TDAH).

\section{Instrumento}

El cuaderno para el proceso de evaluación está formado por:

a) Datos sociodemográficos: edad, sexo, curso de EP.

b) Subpruebas del Test TALE para la evaluación del rendimiento en la escritura (Toro y Cervera, 2009), el cual valora los niveles generales y las características específicas de la lectura y la escritura en un momento concreto del proceso de adquisición de éstas:

- Copia, cuya finalidad es evaluar la capacidad de reproducir la grafia de las palabras.

- Dictado, para la evaluación de la habilidad de convertir fonemas en asociaciones de grafemas. Se considera la coordinación audio-visomotriz correspondiendo a cada nivel de edad un texto.

- Escritura espontánea, donde se deja libertad en la escritura de un párrafo, considerando los aspectos gnoseo-visomotrices. 


\section{Procedimiento}

Se contactó con diversas entidades, tanto centros educativos como con asociaciones, con el fin de obtener una muestra representativa de sujetos con un diagnóstico de TDAH. Una vez seleccionada la muestra, se informó a las familias sobre los objetivos del estudio y firmaron el consentimiento informado correspondiente. A continuación, para la primera fase, se aplicaron las subpruebas seleccionadas del test TALE. Cinco años después, en la siguiente fase, se vuelve a contactar con las familias y con los centros en los que estuvieron escolarizados los alumnos, con el objetivo de volver a evaluarlos.

Las evaluaciones pre y post se realizan en una única sesión, en condiciones idóneas para llevar a cabo la prueba de escritura, controlando los criterios necesarios para una correcta evaluación como, por ejemplo, ajustando la altura de las mesas y las sillas a los participantes. Además, se anotan en todos los apartados observaciones sobre el contexto de la prueba como son el agarre y la postura al escribir.

El análisis de los datos se realiza con el programa estadístico SPSS 24.0. Para el cálculo de las diferencias se calcula la prueba no paramétrica de rangos de Wilcoxon para dos muestras relacionadas y para el cálculo del tamaño del efecto se calcula la fórmula propuesta por Gibbons, Hedeker y Davis (1993) y se interpreta según los criterios propuestos por Cohen (1988).

\section{Resultados}

Los resultados globales obtenidos en la investigación se reflejan en la Tabla 3, mostrando que no se aprecian cambios significativos $(\mathrm{p}<.05)$ en el grupo de participantes con TDAH en ninguna de las variables evaluadas, excepto en tamaño (sig.: .02) con dos casos (13.3\%) con un tamaño de la letra inferior a $2.5 \mathrm{~mm}$, en la primera evaluación, y ocurriendo en 5 casos $(33.3 \%)$ cinco años más tarde (Tabla 4). 
Tabla 3

Resultados de las pruebas pre-post en el grupo con y sin TDAH

\begin{tabular}{|c|c|c|c|c|c|c|c|}
\hline \multirow{3}{*}{ Diagnóstico } & \multirow{3}{*}{ TALE } & \multicolumn{4}{|c|}{ Momento de Evaluación } & \multirow[b]{3}{*}{ Sig. ${ }^{a}$} & \multirow[b]{3}{*}{$\mathrm{TE}^{\mathrm{b}}$} \\
\hline & & \multicolumn{2}{|c|}{ Pretest } & \multicolumn{2}{|c|}{ Postest } & & \\
\hline & & $M$ & DT & $M$ & DT & & \\
\hline \multirow[t]{10}{*}{ TDA/TDAH } & Tamaño & 3 & 1 & 2.20 & 1.08 & .020 & .698 \\
\hline & Irregularidad & .93 & .258 & .80 & .676 & .414 & .197 \\
\hline & Oscilación & 1.33 & .617 & 1.27 & .884 & .782 & .066 \\
\hline & Líneas anómalas & 1.20 & .676 & 1.00 & .845 & .470 & .175 \\
\hline & Interlineación & 1.47 & .640 & 1.00 & .655 & .080 & .484 \\
\hline & Zonas & 1.40 & .632 & 1.07 & .884 & .190 & .324 \\
\hline & Superposición & .07 & .258 & .07 & .258 & 1 & 0 \\
\hline & Soldadura & .07 & .258 & .07 & .258 & 1 & 0 \\
\hline & Curvas & .93 & .799 & .87 & .743 & .792 & .066 \\
\hline & Barras & .47 & .640 & .40 & .632 & .739 & .080 \\
\hline \multirow[t]{10}{*}{ Sin TDA/TDAH } & Tamaño & 2.67 & 1.23 & 1.11 & .333 & .017 & 1.054 \\
\hline & Irregularidad & 1.11 & .333 & .44 & .527 & .034 & .852 \\
\hline & Oscilación & 1.56 & .527 & .56 & .726 & .021 & 1.043 \\
\hline & Líneas anómalas & 1.22 & .667 & .44 & .726 & .080 & .643 \\
\hline & Interlineación & 1.22 & .667 & .44 & .527 & .035 & .844 \\
\hline & Zonas & 1.33 & .500 & .78 & .667 & .034 & .852 \\
\hline & Superposición & 0 & 0 & 0 & 0 & 1 & 0 \\
\hline & Soldadura & 0 & 0 & 0 & 0 & 1 & 0 \\
\hline & Curvas & .44 & .726 & .44 & .726 & 1 & 0 \\
\hline & Barras & .33 & .707 & .22 & .441 & .705 & .108 \\
\hline
\end{tabular}

a. Prueba de rangos con signo de Wilcoxon. b. TE: Tamaño del efecto

Nota: Las significaciones <.05 están en negrita, asi como los tamaños del efecto $>.45$ y que no eran significativos.

Tabla 4

Descriptivos de la variable Tamaño en el año 2014 y 2019 del grupo con TDAH

\begin{tabular}{lcccccc}
\hline & \multicolumn{2}{c}{ Pretest } & & \multicolumn{2}{c}{ Postest } \\
\cline { 2 - 3 } \cline { 6 - 6 } & $\mathbf{n}$ & \% & & n & \% \\
\hline Inferior a 2,5mm & 2 & 13.3 & & 5 & 33.3 \\
Aproximadamente 2,5mm & 1 & 6.7 & & 4 & 26.7 \\
Entre 2,5 y 3,5mm & 7 & 46.7 & & 4 & 26.7 \\
Entre 3,5 y 5mm & 5 & 33.3 & & 2 & 13.3 \\
\hline Total & 15 & 100.0 & & 15 & 100.0 \\
\hline
\end{tabular}


Si tomamos en cuenta el tamaño del efecto, y tomando como criterio de efecto medio (.50), tan sólo la variable interlineación se acerca a dicho valor (.484), en la cual se observa un cambio de 1,47 a 1,00 en el periodo evaluado de cinco años. En esta variable, en la primera evaluación, 8 casos (53.3\%) presentaban distancias muy irregulares y a los 5 años ese número disminuye a 3 (20\%); además, en el primer momento, solo había un caso que mantenía la equidistancia entre las lineas (6.7\%) y a los cinco años son tres casos (20\%) (Tabla 5).

Tabla 5

Descriptivos de la variable Interlineación en el año 2014 y 2019 del grupo con TDAH

\begin{tabular}{lcccccc}
\hline & \multicolumn{2}{c}{ Pretest } & & \multicolumn{2}{c}{ Postest } \\
\cline { 2 - 3 } \cline { 6 - 7 } & $\mathbf{n}$ & \% & & $\mathbf{n}$ & \% \\
\hline Equidistancia entre las líneas & 1 & 6.7 & & 3 & 20.0 \\
Algo irregulares & 6 & 40.0 & & 9 & 60.0 \\
Distancias muy irregulares & 8 & 53.3 & & 3 & 20.0 \\
\hline Total & 15 & 100.0 & & 15 & 100.0 \\
\hline
\end{tabular}

No ocurre así en la muestra de participantes sin TDAH donde se aprecian cambios significativos $(\mathrm{p}<.05)$ en el periodo de cinco años, en el tamaño de la letra (.017) con 7 casos de mejoría (Tabla 6). En la variable Irregularidad o variaciones en el tamaño de las letras (.034) con 5 casos de mejoría (Tabla 7). En la variable Oscilación o grafismo tembloroso (.021) con una mejoría de 5 casos (Tabla 8). En la variable Interlineación o distancia entre las líneas (.035) con 4 casos de mejoría (55.6\%) (Tabla 9). Y las Zonas sobre las que se distribuyen las letras (.034) con 3 casos que mejoran (Tabla 10).

Tabla 6

Descriptivos de la variable Tamaño en el año 2014 y 2019 del grupo sin TDAH

\begin{tabular}{lccccc}
\hline & \multicolumn{2}{c}{ Pretest } & \multicolumn{2}{c}{ Postest } \\
\cline { 2 - 3 } \cline { 5 - 5 } & n & \% & n & \% \\
\hline Inferior a 2,5mm & 1 & 11.1 & 8 & 88.9 \\
Aproximadamente 2,5mm & 4 & 44.4 & 1 & 11.1 \\
Entre 2,5 y 3,5mm & 2 & 22.2 & - & - \\
Entre 3,5 y 5mm & 1 & 11.1 & - & - \\
Superior a 5mm & 1 & 11.1 & - & - \\
\hline Total & 9 & 100.0 & 9 & 100.0 \\
\hline
\end{tabular}


Tabla 7

Descriptivos de la variable Irregularidad en el año 2014 y 2019 del grupo $\sin T D A H$

\begin{tabular}{lcccccc}
\hline & \multicolumn{2}{c}{ Pretest } & & \multicolumn{2}{c}{ Postest } \\
\cline { 2 - 3 } \cline { 5 - 6 } & $\mathbf{n}$ & \% & & n & $\%$ \\
\hline Tamaño homogéneo & - & - & & 5 & 55.6 \\
Leves diferencias de tamaño & 8 & 88.9 & & 4 & 44.4 \\
Grandes diferencias & 1 & 11.1 & & - & - \\
\hline Total & 9 & 100.0 & & 9 & 100.0 \\
\hline
\end{tabular}

Tabla 8

Descriptivos de la variable Oscilación en el año 2014 y 2019 del grupo $\sin T D A H$

\begin{tabular}{lcccccc}
\hline & \multicolumn{2}{c}{ Pretest } & & \multicolumn{2}{c}{ Postest } \\
\cline { 2 - 3 } \cline { 5 - 6 } & $\mathbf{n}$ & $\mathbf{\%}$ & & $\mathbf{n}$ & $\mathbf{\%}$ \\
\hline Grafismo firme & 0 & - & & 5 & 55.6 \\
Ligeramente tembloroso & 4 & 44.4 & & 3 & 33.3 \\
Grafismo muy tembloroso & 5 & 55.6 & & 1 & 11.1 \\
\hline Total & 9 & 100.0 & & 9 & 100.0 \\
\hline
\end{tabular}

Tabla 9

Descriptivos de la variable Interlineación en el año 2014 y 2019 del grupo $\sin T D A H$

\begin{tabular}{lcccccc}
\hline & \multicolumn{2}{c}{ Pretest } & & \multicolumn{2}{c}{ Postest } \\
\cline { 2 - 3 } \cline { 5 - 7 } & $\mathbf{n}$ & $\mathbf{\%}$ & & $\mathbf{n}$ & $\%$ \\
\hline Equidistancia entre líneas & 1 & 11.1 & & 5 & 55.6 \\
Algo irregulares & 5 & 55.6 & & 4 & 44.4 \\
Distancias muy irregulares & 3 & 33.3 & & 0 & - \\
\hline Total & 9 & 100.0 & & 9 & 100.0 \\
\hline
\end{tabular}

Tabla 10

Estadísticos descriptivos de la variable Zonas en el año 2014 y 2019 del grupo $\sin T D A H$

\begin{tabular}{lcccccc}
\hline & \multicolumn{2}{c}{ Pretest } & & \multicolumn{2}{c}{ Postest } \\
\cline { 2 - 3 } \cline { 5 - 6 } & $\mathbf{n}$ & \% & & n & $\%$ \\
\hline Uniformes & 0 & - & & 3 & 33.3 \\
Solo en 2 o 3 ocasiones & 6 & 66.7 & & 5 & 55.6 \\
Muy desiguales & 3 & 33.3 & & 1 & 11.1 \\
\hline Total & 9 & 100.0 & & 9 & 100.0 \\
\hline
\end{tabular}


En la variable Líneas anómalas, la diferencia no es significativa (.08), pero el tamaño del efecto (.643) apunta a un efecto medio-alto. En la evaluación inicial tan solo un caso presentaba líneas continuas/uniformes (11.1\%), y al cabo de cinco años este número es de 6 casos (66.7\%). Por lo tanto, sí que se puede apreciar un cambio positivo (Tabla 11).

Tabla 11

Descriptivos de la variable Líneas anómalas en el momento año 2014 y 2019 del grupo sin TDAH

\begin{tabular}{lccccc}
\hline & \multicolumn{2}{c}{ Pretest } & \multicolumn{2}{c}{ Postest } \\
\cline { 5 - 6 } \cline { 5 - 6 } & $\mathbf{n}$ & $\mathbf{\%}$ & & $\mathbf{n}$ & $\mathbf{\%}$ \\
\hline Continuas uniformes & 1 & 11.1 & 6 & 66.7 \\
Escasas o leves & 5 & 55.6 & 2 & 22.2 \\
Muy frecuentes y acusadas & 3 & 33.3 & 1 & 11.1 \\
\hline Total & 9 & 100.0 & 9 & 100.0 \\
\hline
\end{tabular}

En las variables superposición y soldadura no se observan cambios, ya que todos los casos presentan la máxima valoración en el momento inicial, por lo que la prueba no puede detectar una mejoría en esta variable.

En consonancia con los resultados del test TALE, a medida que el niño está escolarizado en un curso mayor, el grafismo de la escritura tiende a mejorar, pero solamente en la población de participantes sin TDAH.

\section{Discusión y conclusiones}

La hipótesis principal del estudio se demuestra parcialmente ya que no se logra en todas las variables evaluadas. Aunque hay diferencias, a nivel estadístico no se comprueba que sean significativas ni que las distintas subpruebas del TALE arrojen resultados significativos. Sin embargo, Arán y Mías (2009) sostienen que debería ser mejor en la Copia que en el Dictado, ya que los sujetos con TDAH presentan más dificultades para la retención de información verbal y, por lo tanto, la memorización mejora cuando existe un apoyo visual más que auditivo.

Podemos confirmar que, como indican Graham et al. (2006), con la edad, el tamaño de las letras tiende a disminuir y la distancia entre las líneas mejora, pero no podemos confirmar que la formación de la letra, el espacio y el alineamiento tiendan a ser más exactos, ni que la escritura sea más abreviada y la letra cursiva o ligada se desarrolle con curvas que reemplazan a los ángulos y a las ligaduras que unen a las letras. Además, el agarre 
del útil que determina la calidad de la escritura y la postura al escribir, no varían, ya que se adquieren desde los primeros años y es muy complejo modificarlos (Falk, Tam, Schwellnus, y Chau, 2010).

En esta investigación no podemos concluir que la medicación para tratar los síntomas del TDAH pueda mejorar la legibilidad y la precisión como indica Lange et al. (2007), puesto que se aprecia mejora en la escritura de los participantes con TDAH. Hemos comprobado, como afirman Graham et al. (2006) en su investigación, que con la edad, la legibilidad y la velocidad de la escritura progresan, a pesar de que en nuestro estudio se observa que esto sucede solo en los participantes sin TDAH, posiblemente porque la escritura exige múltiples recursos cognitivos (Rodríguez et al. 2010) y se necesitaría su práctica intensiva a lo largo de los cursos escolares (Denton, Cope, y Moser, 2006; Howe, Roston, Sheu, e Hinojosa, 2013), además de entrenamiento en factores neuropsicológicos.

Cinco años después, se ha podido comprobar que los sujetos con TDAH mejoran solamente en las variables tamaño e interlineación, por lo que necesitarian un programa de intervención, mientras que los que no tienen un diagnóstico de TDAH mejoran en su grafía sin ningún tipo de intervención específica. Estos resultados los tomamos con cautela ya que consideramos que son necesarias más investigaciones para determinar las variables asociadas a las dificultades de escritura en sujetos con TDAH y así poder plantear una intervención adecuada. La mayor parte de la investigación en la escritura se ha focalizado, según Fernández-Jaén et al. (2018), en aspectos cualitativos de la letra y basándose en el criterio subjetivo del examinador, no incluyéndose tampoco una medida de seguimiento para determinar la estabilidad del cambio, lo que confiere a nuestro estudio un valor especial.

García-Guirao y Vergara-Moragues, (2015) encuentran diferencias en funcionalidad visual evaluada, a través de los movimientos sacádicos y durante el procesamiento de la información auditiva en sujetos de 7 a 11 años con TDAH. Además, Poeta y Rosa-Neto (2007) observan una dificultad en el control de la postura con un aumento del tono muscular, en la organización espaciotemporal, en el equilibrio, en la motricidad fina y la motricidad global y en el esquema corporal. Por lo que este grupo presenta la necesidad de otro tipo de intervención como, por ejemplo, en habilidades neuropsicológicas, pues según requieren del desarrollo de la organización espacial y destreza 
motora para sostener el útil (Lofiego, 1995, citado en Rosa-Neto et al., 2013), junto con habilidades motrices gruesas y finas, correcta lateralidad y un adecuado desarrollo perceptivo, visual y auditivo.

Por este motivo, Cleary y Scott (2011) abogan por incorporar la neuropsicología clínica a los centros educativos, especialmente cuando existe una base neurológica como en el TDAH, ayudando a comprender los procesos neuropsicológicos del aprendizaje y teniendo en cuenta el valor predictivo de algunas variables neuropsicológicas en el rendimiento escolar. Por ello, creemos conveniente, como indican Fernández-Jaén et al. (2018), que los EOEP realicen estas evaluaciones de cara a plantear una intervención que logre una eficacia motriz en la escritura, ya que la valoración clínica podría ser insuficiente.

No obstante, las pruebas usadas pueden ser interesantes para observar la comorbilidad entre TDAH y dificultades de escritura que, como indican Fernández-Jaén et al. (2018), se da en un alto porcentaje de casos, siendo necesario ampliar los análisis en muestras de mayor tamaño, debido a que el 36\% de los participantes sin TDAH eran niños y en el grupo de participantes con TDAH el $80 \%$ Por consiguiente, en nuestro estudio se confirma, aunque con la cautela de los porcentajes señalados, que el TDAH es más frecuente en los varones como afirma también Rodríguez-Molinero et al. (2009).

Asimismo, consideramos necesarios más estudios longitudinales con instrumentos estandarizados que evalúen áreas distintas, con el objetivo de desarrollar estrategias de intervención más adecuadas ya que, a pesar de que gran parte de la investigación científica está enfocada en el tramo de edad tratado, son escasos los estudios de este tipo que analicen la escritura.

Concluir que este estudio destaca la importancia de la escritura manuscrita que sigue siendo una forma de comunicación y una destreza requerida durante la mayor parte de la jornada escolar. También, tomando los resultados con cautela, podemos ultimar que el desarrollo natural de la habilidad y pericia en la escritura se da con la práctica a lo largo de los años, lo que consigue el aumento de la motivación intrínseca para seguir avanzando, mientras que en los estudiantes de primaria con diagnóstico de TDAH el desarrollo natural queda enlentecido a pesar de los apoyos, lo que puede afectar de forma negativa al sentimiento de eficacia personal. Una escritura inadecuada provoca un decremento de la motivación (Cornhill y Case-Smith, 1996, citado en Lange et al., 2007), una baja autoestima (Brossard-Racine, Majnemer, Shevell, y Snider, 2008) y el alejamiento del éxito académico (Feder y Majnemer, 2007). 


\section{Referencias}

Abad-Mas, L., Ruiz-Andrés, R., Moreno-Madrid, F., Herrero-Lladró, R., y Suay-Nicolás, E. (2013). Intervención psicopedagógica en el Trastorno por déficit de atención/hiperactividad. Revista Neurología, 57(1), 193-203.

https://doi.org/10.33588/rn57S01.2013290

Adi-Japha, E., Landau, Y. E., Frenkel, L., Teicher, M., Gross Tsur, V., y Shalev, S. (2007). ADHD and dysgraphia: underlying mechanisms. Cortex, 43(6), 700-709.

https://doi.org/10.1016/s0010-9452(08)70499-4

Alcover, C. M., y Rodríguez-Mazo, F. (2012). Plasticidad cerebral y Hábito en Williams James: Un antecedente para la neurociencia social. Pshychologia Latina, 3(1), 1-9.

https://doi.org/10.5209/rev_PSLA.2012.v3.u138737

Arán, V., y Mías, C. D. (2009). Neuropsicología del trastorno por déficit de atención/hiperactividad: subtipos predominio Déficit de atención y predominio Hiperactivo-Impulsivo. Revista Argentina de Neuropsicología, 13, 14-28.

Barkley, R. A. (2006). Attention Deficit Hyperactivity Disorder: A handbook for diagnosis and treatment ( $3^{\mathrm{a}}$ ed.). New York: Guilford Press.

Barkley, R. A, y Murphy, K. R. (2005). Attention-deficit hyperactivity disorder: A clinical workbook, Volumen 2. New York: The Guildord Press.

Barkley, R. A, y Murphy, K. R. (2010). Deficient emotional selfregulation in adults with Attention-Deficit/Hyperactivity Disorder (ADHD): The relative contributions of emotional impulsiveness and ADHD symptoms to adaptive impairments in major life activities. Journal of $A D H D \&$ Related Disorders, 1(4), 5-28. https://doi.org/10.1176/appi.aip.2010.10081172

Betegón, E., Rodríguez-Medina, J., e Irurtia, M. J. (2019). Neuroeducación y autocontrol: Cómo vincular lo que aprendemos con lo que hacemos. Un estudio de caso múltiple en Educación Infantil. Revista Interuniversitaria de Formación del Profesorado, 94(33.3), 307-326.

Brossard-Racine, M., Majnemer, A., Shevell, M., y Snider, L. (2008). Handwriting performance in children with attention deficit hyperactivity disorder (ADHD). Journal of Child Neurology, 
23(4), 399-406. https://doi.org/10.1177/0883073807309244

Catalá-López, F., y Hutton, B. (2018). The treatment of attention deficit hyperactivity disorder in children and adolescents: Epidemiology, multimorbidity and integrated health services. Anales de Pediatria, 88(4), 181-182.

https://doi.org/ 10.1016/j.anpede.2017.12.003

Cisneros, N., Lázaro, E. M., García, M. A., García, G., y Quintanar, L. (2012). Aportaciones de la neuropsicología a la evaluación del desempeño escolar y del aprendizaje. Congreso Internacional de Educación. Universidad Autónoma de Puebla, México.

Cleary, M. J., y Scott, A. J. (2011). Developments in clinical neuropsychology: Implications for school psychological servives. Journal of School Health, 81(1), 1-7.

https://doi.org/1111/j.1746-1561.2010.00550.x

Cohen, J. (1988). Statistical power analysis for the behavioural sciences ( $2^{\mathrm{a}}$ ed.). Nueva York: Academic Press.

Cornhill, H., y Case-Smith, J. (1996). Factors that relate to good and por handwriting. American Journal. The American Journal of Occupational Therapy, 50, 732-739.

Denton, P. L., Cope, S., y Moser, C. (2006). The effects of sensoriomotor-based intervention versus therapeutic practiced on improving handwriting perfomance in 6-to 11-year- old children. American Journal of Occupational Therapy, 60, 16-27.

https://doi.org/10.5014/ajot.60.1.16

Díaz-Jara, M. (2016). Procesos y Programas neuromotores y de movimientos rítmicos relacionados con el aprendizaje. En P. Martín Lobo (Coord.), Procesos y programas de neuropsicología educativa (pp. 61-78). Madrid: Ministerio de Educación, Cultura y Deporte: Centro Nacional de Investigación e Innovación (CNIIE).

Falk, T. H., Tam, C., Schwellnus, H., y Chau, T. (2010). Grip force variability and its effects on children's handwriting Legibility, form, and strokes. Journal of Biomechanical Engineering, 132(11), 1-5. https://doi.org/10.5014/ajot.60.1.16

Feder, K. P., y Majnemer, A. (2007). Handwriting development, competency, and intervention. Developmental, Medicine \& Child Neurology, 49(4), 312-317.

https://doi.org/10.1111/j.1469-8749.2007.00312

Fernández-Jaén, A., Fernández-Mayoralas, D. M, FernándezPerrone, A. L., Jiménez, A., Albert, J., López-Martín, S., et al. (2018). Neurodesarrollo y fenocopias del trastorno por déficit de 
atención/hiperactividad: Diagnóstico diferencial. Revista Neurologia, 66(1), 103-107.

https://doi.org/10.33588/rn.66S01.2017532

García-Guirao, E., y Vergara-Moragues, E. (2015). Diferencias entre los movimientos sacádicos, la discriminación auditiva y la lateralidad en niños de 7 a 11 años con y sin trastorno por déficit de atención con y sin hiperactividad. Búsqueda, 14(2), 32-44. https://doi.org/10.21892/01239813.57

García-Sánchez, J. N., y Rodríguez-Pérez, C. (2007). Influencia del intervalo de registro y del organizador gráfico en el procesoproducto de la escritura y en otras variables psicológicas. Psicothema, 19(2), 198-205.

Gibbons, R. D., Hedeker, D. R., y Davis, J. M. (1993). Estimation of effect size from a series of experiments involving paired comparisons. Journal of Educational Statistics, 18(3), 271-279.

https://doi.org/10.2307/1165136

Graham, S., Weintraub, N., y Berninger, V. W. (2006). Hand function in the child. St Louis, Missouri: Mosby Elsevier.

Howard-Jones, P. (2011). Investigación neuroeducativa. Neurociencia, educación y cerebro: De los contextos a la práctica. Madrid: La Muralla.

Howe, T-H., Roston, K. L., Sheu, C-F., e Hinojosa, J. (2013). Assessing handwriting intervention effectiveness in elementary school students: A two-group controlled study. American Journal of Occupational Therapy, 67, 19-27.

https://doi.org/10.5014/ajot.2013.005470

Lange, K. W., Tucha, L. Walitza, S. Gerlach, M. Linder, M., y Tucha, O. (2007). Interaction of attention and graphomotor functions in children with attention deficit hyperactivity disorder. Journal of Neural Transmission, 72, 249-259.

https://doi.org/10.1007/978-3-211-73574-9_31

Lipowska, M. (2012). Graphomotor functions in ADHD-motor or planning deficit? A microgenetic approach. Neuropsychologica, 10(1), 69-80. https://doi.org/10.5604/17307503.995089

Lofiego, J. L. (1995). Disgrafia: Avaliação Fonoaudiológica. Rio de Janeiro: Revinter.

Marambio, C. A. (2017). Estrategias para estimular competencias cognitivas superiores en estudiantes universitarios. Contextos: Estudios de Humanidades y Ciencias Sociales, 38, 81-95. 
Martín Fernández-Mayoralas, D., Fernández Perrone A.L. y Fernández-Jaén, A. (2013). Trastornos específicos del aprendizaje y trastorno por déficit de atención/hiperactividad. Adolescere, 1(2), 69-75.

Martín Lobo, P. (Coord.) (2016). Procesos y programas de neuropsicología educativa. Madrid: Ministerio de Educación, Cultura y Deporte: Centro Nacional de Investigación e Innovación (CNIIE).

Mayes, S. D., Breaux, R. P., Calhoun, S. L., y Frye, S. S. (2017). High prevalence of dysgraphia in elementary through high school students with ADHD and autism. Journal of Attention Disorders, 23(8), 787-796. https://doi.org/10.1177/1087054717720721

Merzenich, M. M, Tallal, P. Peterson, B., Miller, S., y Jenkins, W. M. (1999). Some neurological principles relevant to the origins ofland the cortical plasticity-based remediation of- developmental language impairments. En J. Grafman y Y. Christen (Eds), Neuronal plasticity: Building a bridge form the laboratory to the clinic (pp. 169-187). Berlin, Alemania: Springer-Verlag.

Montoya-Londoño, D. M. Varela-Cifuentes, V., y Dussan-Lubert, C. (2012). Correlación entre las habilidades académicas de lectura y escritura y el desempeño neuropsicológico en una muestra de niños y niñas con TDAH de la ciudad de Manizales. Psicología desde el Caribe, 29(2), 305-329.

Ortiz, T. (2009). Neurociencia y educación. Madrid: Alianza.

Poeta. L. S., y Rosa-Neto, F. (2007). Evaluación motora en escolares con indicadores del trastorno por déficit de atención/hiperactividad. Revista de Neurología, 44(3), 146-149. https://doi.org/10.33588/rn.4403.2005663

Quintanar, L., y Solovieva, Y. (2003). Manual de evaluación neuropsicológica infantil. México: Universidad Autónoma de Puebla.

Razza, R. A., Martin, A., y Brooks-Gunn, J. (2012). The implications of early attentional regulation for school success among lowincome children. Journal of Applied Developmental Psychology, 33(6), 311-319. https://doi.org/10.1016/j.appdev.2012.07.005

Rigal, R. (2006). Educación motriz y educación psicomotriz en Preescolar y Primaria. Barcelona: INDE Publicaciones.

Rivera-Flores, G. W. (2013). Etiología del trastorno por déficit de atención e hiperactividad y características asociadas en la infancia y niñez. Actas de Investigación PsicológicaPsychological Research Records, 3(2), 1079-1091. 
Rodríguez, C., González-Castro, P., Álvarez, L., Cerezo-Menéndez, R., García, J. N., González-Pienda, J. A., Álvarez, D., y Bernardo, A. B. (2010). Un análisis de los modelos teóricos actuales de escritura y su relación con el trastorno por déficit de atención e hiperactividad. Papeles del Psicólogo, 31(2), 211-220.

Rodríguez, C., Álvarez, D., González-Castro, P., García, J. N., Álvarez, L., Núñez, J. C., González, J. A., y Bernardo, A. (2009). TDAH y dificultades de aprendizaje en escritura: comorbilidad en base a la atención y memoria operativa. European Journal of Educational Psychology, 2(3), 181-198.

Rodríguez-Molinero, L., López-Villalobos, J. A., Garrido Redondo, M., Sacristán Martín, A. M., Martinez Rivera, M. T., y Ruiz Sanz, F. (2009). Estudio psicométrico-clínico de prevalencia y comorbilidad del trastorno por déficit de atención con hiperactividad en Castilla y León (España). Revista Pediátrica de Atención Primaria, 11(42), 251-270.

Rosa-Neto, F., Regina, X., Marilia dos Santos, A. P., Nunes-Amaro, K., Florencio, R., y Schilling, L. (2013). Cross-dominance and reading and writing outcomes in school-aged children. Revista CEFAC, 15(4), 864-871.

Sánchez-Pérez, N., y González-Salinas, C. (2013). Ajuste escolar del alumnado con TDAH: Factores de riesgo cognitivos, emocionales y temperamentales. Electronic Journal of Research in Educational Psychology, 11(2), 527-550.

https://doi.org/10.14204/ejrep.30.12189

Stasik, D., Tucha, O., Tucha, L., Walitza, S., y Lange, K. W. (2009). Graphomotor functions in children with attention déficit hyperactivity disorder (ADHD). Psychiatria Polska, 43(2), 183192.

Szucs, D., y Goswami, U. (2007). Educational neuroscience: Defining a new discipline for the study of mental representations. Mind, Brain and Education, 1(3), 114-127.

https://doi.org/10.1111/j.1751-228X.2007.00012.x

Toro, J. y Cervera, M. (2009). Test de análisis de lectoescritura TALE. Madrid: Antonio Machado Libros.

Van Hoorn, J. F., Maathuis, C., Peters, L., y Hadders-Algra, M. (2010). Handwriting, visuomotor integration, and neurological condition at school age. Developmental Medicine \& Child Neurology, 52, 941-947. 
https://doi.org/10.1111/j.1469-8749.2010.03715.x

Volman, M. J. M, Van Schendel, B. M. y Jongmans, M. J. (2006). Handwriting difficulties in primary school children: A search for underlying mechanisms. The American Journal of Occupational Therapy, 60(4), 451-46.

https://doi.org/10.5014/ajot.60.4.451 